\title{
Recalculating interatomic forces
}

\author{
The van der Waals force between atoms and molecules is generally taken to be inversely proportional \\ to the inverse sixth power of the distance, but positronium atoms may interact differently.
}

Although the van der Waals force has been part of classical physics for the best part of a century, since the recognition that the departure of the behaviour of real gases from the perfect gas laws points to a shortrange attractive force between atoms and molecules, there is no classical explanation of the effect. Provided that the interacting species have no permanent dipole moment (when the van der Waals force is negligible), the best that can be done is to suppose that atoms and molecules are attracted to each other as a consequence of their polarizability; each becomes a little dipole under the influence of the other.

Classically, it is easy to see that this force has a short range in the sense that it will be proportional to a large power of the reciprocal distance $R$ between the interacting entities (for the force due to an induced dipole is already proportional to $1 / R^{3}$ ), but two interacting atoms which mutually induce a symmetrical pair of dipoles will be found to have a mutual energy greater than they began with, corresponding to repulsion, and which classically will not happen. That is why the van der Waals force has been regarded as essentially a quantum phenomenon at least since $F$. London's successful account of it half a century ago. Now, even that has been refined by means of an elegant calculation due to J.R. Manson and R.H. Ritchie (Phys. Rev. Lett. 54, 785; 1985). The outcome could be interesting.

The simplest way of calculating the van der Waals force gives a reasonable result, but is now shown to be subtly too simple. For definiteness, suppose that the two interacting atoms are two hydrogen-like atoms whose nulcei are separated by a distance $R$. To a first approximation, the single electrons of each of them are described by a set of hydrogen-like wavefunctions centred on points separated by a distance $R$. The more realistic next approximation then consists in the addition to the total energy of the system of the terms representing the interaction between the pair of nuclei and the pair of electrons (both positive, denoting repulsion) and between each nucleus and the other electron (negative, for attraction).

Practitioners of wave mechanics will take this kind of problem in their stride. The extra interaction energy is supposed small compared with the interaction between each nucleus and its "own" electron, so textbook perturbation theory applies. The complete set of wavefunctions of each atom enters into some linear combination of the wavefunctions corresponding to all of its quantum states. London remarked that geometrical symmetry ensures that the firstorder perturbation calculation is zero. But second-order perturbation theory yields a plausible result: an attractive force between atoms proportional to $1 / R^{6}$.

At its simplest, perturbation theory is a way of calculating the energy of a system that differs to only a small extent from one that can be solved exactly. For the calculation of van der Waals force between two hydrogen-like atoms, for example, the behaviour of each electron under the influence of its own nucleus is exactly calculable, and the result is the familiar infinite set of discrete energy levels converging on the ionization energy of the atom. The energy of the combined system of two atoms close together is then approximately calculable from the terms representing the interaction between the atoms and the wavefunctions corresponding to the original unperturbed system, the product of two one-electron wavefunctions centred on the positions of the two nuclei separated by a specific distance.

Second-order calculations give a familiar result. The energy of the interacting system differs from that in which the interaction is ignored by an amount expressed as an infinite series in which each term represents the square of the probability that the interaction energy will induce a transition from the original state to another but weighted by the reciprocal of the energy difference between the states. Straightforward calculation along these lines recovers the London result that the energy of the combined system is decreased by an amount proportional to $1 / R^{6}$, entirely appropriate for the short-range van der Waals force.

But the argument is incomplete. Implicit in the calculation is the assumption that the pair of interacting atoms is static, with the two nuclei fixed in space. Yet the calculation itself suggests that the interaction is responsible for an attractive force between the pair of atoms which in the absence of specified constraints, should be sufficient to make the configuration of the system time-dependent.

The circumstances resemble those familiar in the calculation of the properties of molecules, where it is customary to calculate electronic states on the assumption that the atomic nuclei are fixed in space, and then separately to calculate such things as the vibrational frequencies of the molecule, which entails allowing that the nuclei can move. The justification of this is the Born-Oppenheimer approximation, which is usually sustained by the observation that the characteristic frequencies of electronic motions are much greater than those with which atomic nuclei move. One day some lucky graduate student will find a supervisor to back sober up-to-date investigation of the circumstances under which the approximation does not apply.

Meanwhile, Manson and Ritchie have provided an intriguing example with which to begin, the van der Waals interaction between two positronium atoms, the metastable atoms consisting of a positron and an electron, the least massive of all hydrogen-like atoms. The starting-point for the calculation is a description of the unperturbed state of the system which allows that each of the interacting atoms may be in motion (so the wavefunctions are products of two atomic wavefunctions and two momentum wavefunctions, or plane waves). What Manson and Ritchie have done is to calculate the van der Waals force between two atoms whose initial states are described in this way.

First, there is a matter of interpretation, which goes back to London. The terms in the series representing the second-order perturbation energy, which are products of the probability that the interaction energy will cause transitions between electonic states, may be regarded as representing processes in which a virtual photon is allowed to excite the system in a defined way and then be emitted again, to be absorbed where it began. But, in such a process, there must be not merely a transfer of energy but of momentum - the atoms concerned must recoil. Manson and Ritchie have allowed for recoil in the calculation of the forces between positronium atoms. So the $1 / R^{6}$ behaviour of the van der Waals force is modified, by the effects of recoil, to one that tends to $1 / R^{4}$ at small separations between the atoms.

The chief interest in this work will be the neatness of the calculation. For the interaction between positronium atoms, the departure from $1 / R^{6}$ behaviour should be recognizable out to a distance of four atomic units, and may thus be measurable. So should be departures from London's result for the interaction between positronium and other atoms. Meanwhile, the calculation is a telling reminder that even the best-tried algorithms should be constantly reassessed.

John Maddox 